\title{
Universal correlation between CP phase $\delta$ and the non-unitarity $\alpha$ parameter phases
}

\author{
Hisakazu Minakata*† \\ Instituto Física Teórica, UAM/CSIC, Calle Nicola's Cabrera 13-15, Cantoblanco E-28049 \\ Madrid, Spain \& Research Center for Cosmic Neutrinos, Institute for Cosmic Ray Research, \\ University of Tokyo, Kashiwa, Chiba 277-8582, Japan \\ E-mail: hisakazu.minakata@gmail.com
}

\section{Ivan Martinez-Soler}

Theoretical Physics Department, Fermi National Accelerator Laboratory, P.O. Box 500, Batavia IL 60510, USA \& Department of Physics and Astronomy, Northwestern University, Evanston, IL 60208, USA \& Colegio de Física Fundamental e Interdisciplinaria de las Américas (COFI), 254 Norzagaray street, San Juan, Puerto Rico 00901

E-mail: ivan.martinezsoler@northwestern.edu

The non-unitarity of the neutrino mixing matrix is a problem related with a more fundamental question about the origin of the neutrino mass. After a brief discussion on the questions "why do we need model-independent framework for unitarity test?", we show interesting properties present in the oscillation probabilities of neutrinos propagating in matter with non-unitarity. That is, (1) partial unitarity and (2) universal phase correlation. To illuminate these points, we formulate a perturbative framework with the two expansion parameters $\varepsilon \equiv \Delta m_{21}^{2} / \Delta m_{31}^{2}$ and $\alpha$ matrix elements. The complex triangular $\alpha$ matrix is introduced through the definition of $3 \times 3$ non-unitary flavor mixing matrix $N$ as

$$
N=(\mathbf{1}-\alpha) U=\left\{\mathbf{1}-\left[\begin{array}{ccc}
\alpha_{e e} & 0 & 0 \\
\alpha_{\mu e} & \alpha_{\mu \mu} & 0 \\
\alpha_{\tau e} & \alpha_{\tau \mu} & \alpha_{\tau \tau}
\end{array}\right]\right\} U
$$

where $U$ is the unitary MNS mixing matrix, and hence $\alpha$ characterizes the size and the flavor dependence of unitarity violation (UV) ${ }^{\ddagger}$ caused by new physics (NP) at low- or high-scales.

The point (1) above essentially means that despite non-unitary mixing, neutrino evolution must be unitary because the three active neutrinos span a complete state space of neutral leptons. The phase correlation mentioned in the point (2) refers an intriguing property that the complex $\alpha$ parameters and $v$ Standard Model CP phase $\delta$ always come into the oscillation probabilities in a correlated way, $e^{-i \delta} \alpha_{\mu e}, \alpha_{\tau e}$, and $e^{i \delta} \alpha_{\tau \mu}$, universally in all the oscillation channels. The physical meaning of this result is briefly discussed.

The 20th International Workshop on Neutrinos (NuFact2018)

12-18 August 2018

Blacksburg, Virginia

\footnotetext{
* Speaker.

${ }^{\dagger}$ Address after February 2019: Center for Neutrino Physics, Virginia Tech, Blacksburg, Virginia

‡Despite that in the physics literature UV usually means "ultraviolet", we use UV in this manuscript as an abbreviation for "unitarity violation" or "unitarity violating".
} 


\section{Model-independent unitarity test}

Probably, the first question one asks in thinking about unitarity test in the lepton sector would be: Given that it is difficult to construct the "MNS triangle", how and in what circumstance do we carry out leptonic unitarity test? We must anticipate that unitarity test is to be carried out without knowing what is NP. If we know what it is, we can just go to models of the NP to confront them to experiments! Then, the only way we could proceed is to prepare (as much as possible) modelindependent framework for unitarity test. A version for low-scale UV is described in refs. [1,2]. In the rest of this manuscript we discuss low-scale UV in its leading order, or high-scale UV.

\section{Correlation between $\mathrm{CP}$ phase $\delta$ and the unitarity violating $\alpha$ parameter phases}

Following the previous effort and to understand the physics of UV, we went on to construct a perturbation theory of UV effect in matter [3], whose outcome will be briefly reported in this manuscript. We have used the two expansion parameters, $\varepsilon \equiv \Delta m_{21}^{2} / \Delta m_{31}^{2}$ (helio-to-terrestrial ratio) and the $\alpha$ matrix elements, while we treat the effects of the matter potential and of $\theta_{13}$ to all orders. We have derived the formulas of the oscillation probabilities to first order in $\varepsilon$ and $\alpha$ 's.

We find that the oscillation probabilities can be decomposed into the three terms (with superscript indicating the order of expansion) as

$$
P\left(v_{\beta} \rightarrow v_{\alpha}\right)=P\left(v_{\beta} \rightarrow v_{\alpha}\right)_{\text {helio }}^{(0+1)}+P\left(v_{\beta} \rightarrow v_{\alpha}\right)_{\text {int-UV }}^{(1)}+P\left(v_{\beta} \rightarrow v_{\alpha}\right)_{\text {ext-UV }}^{(1)},
$$

where the first term is the $v \mathrm{SM}$ mixing part given in [4], while the second term exhibits the effect of (must be) unitary evolution of neutrinos. The third term, which is special to UV scenario, represents the effect of non-unitarity which comes from initial projection onto the mass eigenstate and final projection back to the flavor state using the non-unitary mixing matrix. Hence, the only

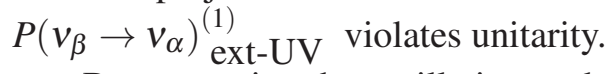

By computing the oscillation probabilities to first order in the expansion parameters, we have demonstrated a very interesting property of universal phase correlation: The complex $\alpha$ parameters come in into the oscillation probabilities always in combination with $\nu \mathrm{SM} \mathrm{CP}$ phase $\delta$ as $e^{-i \delta} \alpha_{\mu e}$, $\alpha_{\tau e}$, and $e^{i \delta} \alpha_{\tau \mu}$ in all the oscillation channels [3]. What do these results mean?

- The framework of UV itself somehow places a gentle constraint on NP that causes nonunitarity by relating the complex UV parameters $\alpha$ 's to the $v$ SM CP phase $\delta$.

- Partial unitarity is a reflection of the completeness of neutral lepton space spanned by the three active neutrinos.

\section{References}

[1] C. S. Fong, H. Minakata and H. Nunokawa, JHEP 1702 (2017) 114 doi:10.1007/JHEP02(2017)114 [arXiv:1609.08623 [hep-ph]].

[2] C. S. Fong, H. Minakata and H. Nunokawa, arXiv:1712.02798 [hep-ph].

[3] I. Martinez-Soler and H. Minakata, arXiv:1806.10152 [hep-ph].

[4] H. Minakata and S. J. Parke, JHEP 1601 (2016) 180 doi:10.1007/JHEP01(2016)180 [arXiv:1505.01826 [hep-ph]]. 